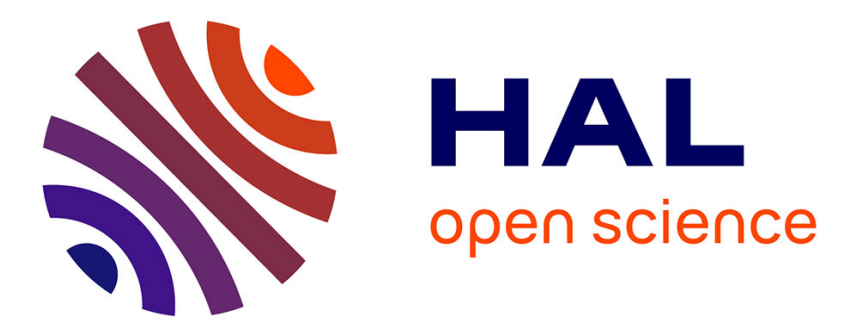

\title{
La montée en autonomie des équipes pédagogiques
}

Baby Lenhard, Audrey Murillo

\section{To cite this version:}

Baby Lenhard, Audrey Murillo. La montée en autonomie des équipes pédagogiques. Jean-François Marcel. Lycées agricoles en changement. Regards pluriels, 2014. halshs-03087158

\section{HAL Id: halshs-03087158 \\ https://shs.hal.science/halshs-03087158}

Submitted on 7 Jan 2021

HAL is a multi-disciplinary open access archive for the deposit and dissemination of scientific research documents, whether they are published or not. The documents may come from teaching and research institutions in France or abroad, or from public or private research centers.
L'archive ouverte pluridisciplinaire $\mathbf{H A L}$, est destinée au dépôt et à la diffusion de documents scientifiques de niveau recherche, publiés ou non, émanant des établissements d'enseignement et de recherche français ou étrangers, des laboratoires publics ou privés. 


\title{
LA MONTÉE EN AUTONOMIE DES ÉQUIPES PÉDAGOGIQUES
}

\author{
Baby Lenhard, Audrey Murillo
}

in Jean-François Marcel, Lycées agricoles en changement

Éducagri éditions | «Agora »

2014 | pages 43 à 61

ISBN 9782844449788

Article disponible en ligne à l'adresse :

https://www.cairn.info/lycees-agricoles-en-changement---page-43.htm

Distribution électronique Cairn.info pour Éducagri éditions.

(C) Éducagri éditions. Tous droits réservés pour tous pays.

La reproduction ou représentation de cet article, notamment par photocopie, n'est autorisée que dans les limites des conditions générales d'utilisation du site ou, le cas échéant, des conditions générales de la licence souscrite par votre établissement. Toute autre reproduction ou représentation, en tout ou partie, sous quelque forme et de quelque manière que ce soit, est interdite sauf accord préalable et écrit de l'éditeur, en dehors des cas prévus par la législation en vigueur en France. Il est précisé que son stockage dans une base de données est également interdit. 


\title{
La montée en autonomie des équipes pédagogiques
}

\author{
Baby LENHARD, professeure d'anglais en lycée agricole \\ Audrey MURILLO, maître de conférences en sciences de l'éducation, \\ université de Toulouse, ENFA, UMR EFTS
}

APPROPRIATION, AUTONOMIE, COLLECTIF D'ENSEIGNANTS, ÉQUIPE PÉDAGOGIQUE, PRESCRIPTION, PROJET

\section{Introduction}

Depuis plus d'un demi-siècle, de multiples réformes tant au niveau structurel (décentralisation, autonomie des établissements, professionnalisation des enseignants) que curriculaire (approche par compétences, pluridisciplinarité, accompagnement personnalisé) ont eu pour effet une modification conséquente de la nature du travail enseignant. Si la transmission des savoirs demeure encore au centre de la fonction, d'autres objectifs sont venus s'y ajouter, comme la formation à l'esprit critique ou la préparation à la vie citoyenne et professionnelle. Pour ce faire, les prescripteurs incitent à privilégier une pédagogie de projet par rapport au face-à-face traditionnel. C'est ainsi que les programmes s'orientent autour de blocs d'enseignement appelés modules, au sein desquels le regroupement de plusieurs disciplines vise des objectifs de transversalité, de progression commune et d'évaluation transdisciplinaire. Cependant, si la modularité (regroupement de plusieurs disciplines dans un module) introduisant la pluridisciplinarité n'est pas un changement récent ${ }^{18}$, elle s'est toutefois étendue depuis les années 1960 à tous les niveaux d'enseignement dans les lycées agricoles. Dans l'esprit des prescripteurs, la pluridisciplinarité a aussi pour objectif de rendre l'apprentissage plus cohérent en favorisant une approche moins atomisée des savoirs: les contenus disciplinaires sont appréhendés au travers des angles multiples, scientifique, technique et culturel, dont l'association permet de présenter une vision nuancée et plus complexe du monde.

Or, le changement porté par la modularité affecte l'architecture des formations et leurs contenus, mais il favorise également le développement du travail en équipe, rendu

18. La modularité apparaît pour la première fois en 1965 dans les lycées agricoles. 
désormais nécessaire pour effectuer la mise en œuvre de l'enseignement modulaire. Dans cette présentation, nous approchons le changement organisationnel selon plusieurs axes: - sur un plan onto-artefactuel, nous décrivons d'abord ce que pensent et font les sujets face à l'artefact (la nouvelle prescription);

- nous nous penchons ensuite sur les sujets lors d'un retour sur l'action (plan ontopraxique) mais d'un point de vue réflexif;

- enfin, nous analysons comment se transforme l'artefact au contact de l'action des sujets (plan arte-praxique).

Cependant, si nous parvenons sans peine à identifier la source première du changement (incrémental car datant de la rénovation du brevet de technicien agricole en 1965), sa temporalité est en revanche moins aisée à définir puisque le produit se trouve en flux constant (Alter, 2003a), affecté par d'autres composants entrant constamment en interrelations et autour desquels évolue l'organisation: les acteurs (axe ontologique), leurs actions (axe praxique), les produits (axe artefactuel), et la connaissance (axe épistémologique). Concrètement, suite au premier dispositif de modularité, d'autres prescriptions sont venues se rajouter depuis, toutes allant dans le même sens et renforçant ainsi le principe de la pluridisciplinarité comme étant au centre de l'architecture des formations dans les lycées agricoles. C'est ainsi que ce changement en continu favorise à terme un développement du travail en équipe jusqu'à une reconfiguration du métier d'enseignant (Marcel, 2006).

Plusieurs interrogations se posent à nous: comment les enseignants réagissent-ils face à la prescription? Pourquoi et comment se manifeste la résistance face à la nouvelle norme ou à son appropriation? Comment interprètent-ils et mettent-ils en œuvre les nouvelles prescriptions guidant leur travail? Quel est l'impact de l'axe artefactuel du changement (imposition de la prescription) sur la constitution et la pérennisation des équipes pédagogiques?

Ces questions nous amènent à enquêter sur le travail de trois équipes pédagogiques dans un établissement d'enseignement agricole. En partant, dans chaque cas, d'une lecture préalable de la prescription, nous examinons à travers une appréciation individuelle du travail réalisé au sein du collectif comment les membres perçoivent le changement et agissent sur lui. Nous nous attardons particulièrement sur la question de l'autonomie du groupe avec une question sous-jacente: qu'est-ce qui détermine la capacité d'agir d'une équipe pédagogique et quels sont les facteurs favorisant la construction d'une nouvelle autonomie?

Dans une première partie, nous nous interrogeons sur la nature des rapports entre le changement prescrit et sa mise en œuvre. En d'autres termes, nous analysons le rapport entre la prescription institutionnelle et l'activité des enseignants. Nous utilisons un cadre conceptuel basé sur l'ergonomie de l'activité (Six, 1999; Clot, 1999) pour mener cette 
réflexion. Notre deuxième partie est consacrée à la présentation des collectifs d'enseignants choisis pour la variété des prescriptions caractérisant leur activité, ainsi qu'à l'analyse de l'organisation au sein de laquelle ils évoluent. Enfin, notre dernière partie présente les résultats de l'enquête, avec une analyse comparative des rapports entre tâche, activité et autonomie. Nous évaluons particulièrement la marge d'autonomie variable suivant le type de collectif et tentons d'en expliquer les raisons. Nous tentons aussi de relever les constances et variations dans l'appropriation de la nouvelle norme et la résistance face à elle.

\section{Le travail enseignant entre prescription et activité}

Au sens ergonomique, la prescription (ou tâche prescrite) représente la règle ou la norme à appliquer pour pouvoir réaliser une activité. L'activité est donc toujours régie par la prescription qui permet d'organiser le travail tout en harmonisant les façons de faire. La tâche prescrite représente ce qui est à faire, et l'activité, ce qui se fait (Montmollin, 1986). Malgré son caractère normatif, une prescription ne peut pas être respectée à la lettre, une application mécanique du texte étant impossible dans le travail humain. En effet, la prescription doit être interprétée avant d'être réalisée, ce qui permet à l'opérateur de se saisir de l'objet travail pour l'ordonner à sa façon et de se l'approprier. Ainsi, même lorsqu'elle est accompagnée de cadrages précis, il existera toujours un écart entre prescription et activité (Leplat et Hoc, 1983). Cet écart, pourtant, loin d'être une anomalie, est au contraire considéré comme bénéfique pour le travailleur, car il représente pour les ergonomes l'expression de son autonomie. Dans le cadre de l'enseignement, cela expliquerait les différences entre un collectif de travail et un autre sur des prescriptions identiques.

\subsection{L'activité enseignante est cadrée par la prescription}

Les prescriptions se distinguent par leur degré de cadrage: la prescription discrétionnaire (Maggi, 1996) décline des objectifs sans préciser les manières de les réaliser, et la taylorienne (Pastré, 2007) décrit des prescriptions très précises qui guident pas à pas l'activité en incluant ses modalités de réalisation.

Dans une organisation scolaire, les documents prescriptifs guidant la mise en œuvre des enseignements et évaluations sont les programmes ou référentiels (lycées agricoles). Chez les enseignants, ils sont loin d'être simplement un ensemble de directives et d'injonctions guidant leur travail, et possèdent au contraire une sorte de mémoire collective de travail (genèse instrumentale, Rabardel, 1995), fournissant un repère essentiel dans l'effort d'interprétation et de mise en œuvre de la tâche. C'est pourquoi Six (1999) propose de s'intéresser aux prescripteurs et aux concepteurs pour mieux comprendre l'activité réelle. Reprenant une proposition de Clot (1995), il avance l'idée de tâche prescrite comme 
« modèle refroidi » de l'activité des prescripteurs, et considère la tâche comme un processus et non comme un état. Ainsi, tâche et activité sont fortement liées, et sa réflexion sur le travail des cadres l'amène à conclure que ceux-ci sont aussi des prescripteurs. Tout comme pour les cadres, l'action collective des enseignants consiste à faire correspondre leur action avec ce qui a été prévu. Mais face à la variabilité des situations de travail, le collectif devient l'origine d'une transformation importante de la prescription en construisant des situations nouvelles ou inédites (prescription remontante). Nous partons d'un a priori que le travail collectif, par sa nature même, est intrinsèquement lié à la création d'une marge d'autonomie. C'est pourquoi notre intérêt principal porte sur la prescription elle-même (l'artefact ou le produit de l'organisation) et sur la manière dont les équipes réagissent face à elle (plan onto-artefactuel), et, dans le cadre d'une auto-prescription, aux prescripteurs eux-mêmes.

Ainsi, comme mentionné dans l'introduction de cet ouvrage, nous constatons une interrelation entre le plan onto-artefactuel (les liens entre la prescription et la réaction des acteurs) et le plan arte-praxique (I'évolution du produit et la transformation de l'artefact par l'action des acteurs) suivant le sentiment qu'éprouvent les acteurs, entre contrainte et approbation, lesquelles peuvent se traduire soit par le retrait ou l'engagement, ce qui influence en retour l'évolution du produit.

L'activité, le produit d'un travail d'appropriation de la prescription, est en réalité constituée de plusieurs phases distinctes:

- face à la prescription, l'opérateur commence par l'interpréter pour lui donner un sens, ce qui lui permet d'organiser l'action;

- s'ensuit une phase d'anticipation et de préparation de l'activité où tous les paramètres sont considérés, évalués et mis à profit pour la réussite de l'action;

- ensuite, c'est le déroulement de l'activité réelle représentant la mise en œuvre de la prescription;

- enfin, a lieu une étape réflexive, conscientisée ou non, où la scène est rejouée mentalement pour en évaluer les forces et faiblesses. Cette dernière phase contient aussi ce que Clot (1999) appelle le non-réalisé correspondant aux possibles de l'activité. Elle peut être féconde en fournissant des indices à l'opérateur pour améliorer l'activité réelle. Ainsi, l'activité consiste toujours en une renormalisation de la prescription (Schwartz, 1992) découlant d'une nécessaire adaptation de la tâche à la situation du travailleur. Chez les enseignants particulièrement, la cyclicité du rythme scolaire les engage dans un éternel recommencement. De fait, les mises en œuvre de la tâche (sauf dans le cas d'une tâche nouvelle) correspondent souvent à des re-mises en œuvre.

Nous soumettons une définition de l'activité collective à la suite de Leplat (1993), qui opère une distinction entre une «équipe formelle» de travail dont les membres fonctionnent sur la base d'une organisation identifiée et stabilisée à travers le temps, et une «équipe effective» qui se regroupe pour réaliser un travail collectif. L'auteur qualifie 
l'activité collective comme "l'exécution d'une tâche » qui «entraîne l'intervention coordonnée de plusieurs opérateurs» (Leplat, 1993, p. 10), tout en soulignant l'aspect essentiel de la notion d'interdépendance entre les opérateurs. L'activité collective est ainsi une action conjointe des opérateurs partageant un objectif commun. À l'instar de Marcel (2006), nous établissons ainsi une différence entre équipe pédagogique d'un établissement scolaire (équipe formelle) et collectif de travail (équipe effective).

\subsection{Le travail collectif engendre un nouvel espace d'autonomie}

La plupart des recherches sur le travail enseignant en milieu scolaire se sont centrées sur les activités en classe, avec le constat d'une prédominance de la dimension cellulaire du métier, évoquée pour la première fois par Lortie (1975) à travers l'image de la structure alvéolaire des salles de classe. Le sociologue parle ainsi du système scolaire moderne comme d'une organisation construite autour de la segmentation des disciplines d'enseignement au lieu d'une interdépendance. Pourtant, le travail enseignant n'est aujourd'hui plus marqué par le seul périmètre des activités pédagogiques en classe, et cet aspect solitaire autrefois prédominant se modifie pour incorporer des pratiques collectives de plus en plus visibles (Piot, Marcel et Tardif, 2009). Celles-ci permettent au groupe de créer un nouvel espace d'autonomie. L'autonomie n'est cependant pas un objectif en soi. L'autonomie professionnelle chez les enseignants est de nature pédagogique en ce sens qu'elle fournit aux équipes l'occasion de planifier elles-mêmes la conception et la progression des enseignements et ses modalités de mises en œuvre. De façon traditionnelle, l'enseignant a toujours bénéficié d'une grande autonomie dans sa profession et connaît peu de contraintes à l'intérieur des murs de sa classe.

L'autonomie collective est toutefois de nature autre puisque, d'une part, elle s'exerce surtout en dehors de la classe, et, d'autre part, elle met en jeu des compétences nouvelles dues à une gestion simultanée de plusieurs paramètres (organisation, gestion et mise en œuvre coordonnée et synchronique des enseignements). L'autonomie collective, abondamment traitée dans la littérature spécialisée en particulier anglo-saxonne ${ }^{19}$, implique une liberté de conception, la convergence d'objectifs, un faible niveau de contrôle, et une relation d'interdépendance par rapport à la tâche. Elle concerne à la fois la programmation (conception et planning des échéances) et la réalisation (coordination, mise en œuvre et exploitation didactique). Plusieurs études (Cohen et Bailey, 1997 ; Campion, Medsker et Higgs, 1993) ont montré l'existence d'un lien entre des groupes de travail autonomes ou semi-autonomes et l'efficacité de travail. La planification et la coordination favoriseraient le développement de certaines compétences spécifiques et des conduites réflexives propres à l'activité collective des enseignants telles que l'anticipation, la gestion de l'imprévu et le traitement renouvelé de l'inconnu ou de l'incertitude. Nous pensons que l'autonomie résultant de l'appropriation de la tâche signifie bien plus que la seule liberté de conception

19. Paul et Robertson, 1970 ; Campion, Medsker et Higgs, 1993. 
et de mise en œuvre des apprentissages. Elle est en fait indissociable de l'activité réelle et de la façon dont les enseignants parviennent à s'approprier la prescription et à créer leur propre style (Clot, 1999). En d'autres termes, l'espace d'autonomie contient la marque du collectif et s'y développe un apprentissage à la fois social et professionnel.

\section{Une enquête auprès de trois équipes d'enseignants}

Nous avons mené une enquête qualitative dans un lycée agricole durant une année scolaire entière et suivi trois équipes d'enseignants durant les phases de programmation et de mise en œuvre de l'activité collective. Deux séries d'entretiens individuels semi-directifs ont été réalisées, au début et à la fin de l'activité collective. Nous avons pris en compte plusieurs paramètres: perception de la tâche, examen des documents de planification de l'activité, perception de l'activité réelle et enfin perception a posteriori de l'activité. Nous avons aussi observé les temps formels de concertation (réunions de filières, conseil de classe, réunions plénières) pour connaître les modalités de constitution des équipes, et les moments informels (échanges entre membres) pour apprécier l'ambiance de travail. Dans le même temps, nous avons procédé à une lecture systématique des référentiels pour connaître les attentes des prescripteurs, version ensuite croisée avec l'interprétation qu'en font les enseignants. Enfin, nos données ont été traitées selon le modèle de flux de Miles et Huberman (2003).

\subsection{Analyse de l'organisation: des caractéristiques favorables à la pérennisation de l'activité collective}

La sociologie organisationnelle de Mintzberg (1982) nous permet de dégager les grands traits de la structure étudiée afin de comprendre sa dynamique collective. L'établissement présente une «configuration hybride » avec un aspect «professionnel» dominant, et des aspects secondaires, qualifiés $d^{\prime}$ " adhocratique » et de «missionnaire». La "configuration professionnelle » comprend majoritairement du personnel qualifié ayant des compétences spécifiques, l'adhocratique se distingue par un « ajustement mutuel » réalisé sous la forme d'échanges informels entre agents, et la missionnaire procède par «standardisation des normes» en accentuant les représentations communes.

Sous son aspect adhocratique, nous remarquons le rôle socialisant des espaces communs (cantine scolaire, salle des professeurs, couloirs, salle des photocopieuses), où les enseignants échangent sur des sujets variés et parfois personnels, sur les classes et les élèves, et coordonnent le travail collectif. Ce sont des espaces régulateurs de l'activité professionnelle, créateurs de normes et favorisant la cohésion. 
Comme dans une configuration professionnelle ou adhocratique, les enseignants observés bénéficient d'une grande autonomie de travail avec une division verticale et horizontale relativement faible. Cette autonomie est cependant soumise à une guidance pédagogique concertée entre direction et coordonnateurs. Par exemple, l'établissement a connu, avec I'introduction des EIE 20 en 2009, un engouement sans précédent pour les projets collectifs, mais l'envergure de la participation avec le risque de redondance thématique a fini par brouiller la lisibilité des actions. Trois diagnostics principaux furent établis par la direction et les coordonnateurs: a) il y a trop de "petits» projets, entraînant une dispersion de l'énergie collective; b) les élèves sont "perdus» dans cette multitude et remettent en cause la prédominance des matières générales au détriment des matières techniques; c) certains projets demandent à être mieux formulés, en lien avec les objectifs des référentiels afin d'augmenter leur cohérence. Les trois points furent traités l'année suivante: d'abord, les projets sont passés de 14 à 6 , certains collectifs ont fusionné leurs problématiques pour offrir des propositions plus lisibles aux yeux des élèves, et la technique fut remise au centre. Cette démarche de régulation fut déjà opérée par la direction et les coordonnateurs, et validée ensuite par l'ensemble des équipes pédagogiques.

Comme dans une configuration missionnaire, l'établissement présente une forte adhésion aux valeurs communes (standardisation des normes) et aux buts de mission (les objectifs communs). Le projet d'établissement de 2011 nous informe que les valeurs partagées sont celles liées au collectif: «[...]le fort sentiment d'attachement à l'intérêt général qui a permis de résister aux multiples tensions qui ne manquent pas d'affecter la vie d'un établissement [...]. L'identité qui s'est clairement forgée au fil des deux dernières décennies possède encore aujourd'hui un fort pouvoir de cohésion».

La façon dont les équipes ont investi le nouvel espace d'autonomie (EIE) montre le potentiel de dynamique collective existant au sein de l'établissement. Par ailleurs, notre observation des espaces socialisants nous amène à penser que ceux-ci jouent aussi pleinement leur rôle de régulation, tout comme la direction dont la présence active sert à corriger des trajectoires flottantes et à redynamiser les équipes.

\subsection{Présentation des trois équipes}

Notre enquête est basée sur une analyse multi-cas, avec un échantillon composé de trois équipes d'enseignants représentant une «variation maximale ( Miles et Huberman, 2003), c'est-à-dire que l'activité de chacune est caractérisée par un degré de cadrage différent, allant d'une prescription délimitant de très près l'activité (collectif 1 ) jusqu'à une autoprescription (collectif 3) en passant par une version hybride (collectif 2).

20. EIE : espace d'initiative locale, module issu de la réforme du bac professionnel représentant la marge d'autonomie de l'établissement sur une partie du curriculum et contribuant à transférer en partie la responsabilité de la prescription aux équipes d'enseignants. 
Le collectif 1 est composé de quatre enseignants intervenant dans les disciplines du module M22 ${ }^{21}$ en BTSA: français (F1)22, documentation (D1), et ESC (ESC1). L'économie n'intervient que ponctuellement (E1) durant les plages pluridisciplinaires. L'objectif général du module est d' « améliorer ses capacités de recherche et de traitement de l'information, ses capacités d'expression, de communication, de relation et d'initiative » (Document d'accompagnement, 2009). Les disciplines présentes sont non seulement complémentaires, mais elles sont contraintes par des évaluations communes ( $\mathrm{CCF}^{23}$ de français et de documentation et épreuve terminale 1 en commun) et l'utilisation de supports liés au thème culturel au programme ${ }^{24}$. Comme le souligne le prescripteur, le M22 « est un module ouvertement interdisciplinaire». L'équipe met ainsi en œuvre une activité entièrement cadrée par le prescripteur.

Quant au collectif 2, il regroupe trois enseignants autour du module MG125: ESC (ESC2), français histoire-géographie (F2) et documentation (D2), dont le travail collectif (projet P.H) sert aux évaluations certificatives et représente l'aboutissement d'un travail pluridisciplinaire sur une année scolaire. Le MG1 se caractérise par une prescription mixte, en partie guidée (documentation) et en partie libre (français, histoire-géographie, ESC). Les enseignants peuvent ainsi interpréter la prescription de manière à convenir aux besoins du contexte. La collaboration se caractérise en grande partie par du volontariat, car la contrainte d'une évaluation certificative en lien avec une autre discipline ne s'applique que pour la documentation. Cependant, I'ESC utilise aussi le projet comme support de CCF.

Enfin, le collectif 3 porte bien son nom de collectif puisque le groupe se forme spontanément à partir d'une proposition de projet émanant des membres pour la classe de BTSA. Le projet est en rapport avec la formation et s'inspire du référentiel mais n'a aucun caractère obligatoire. Le collectif, composé d'un noyau dur de trois enseignants (économie, E3; technique, T3-1; technique, T3-2), intègre toutefois toutes les disciplines et met en œuvre un projet de conception et de réalisation en aménagement paysager.

En résumé, nous présentons ci-après, sous forme de tableau, les trois équipes en précisant le type de collectif et de prescription, et les objectifs des modules.

21. Module M22 en BTSA (brevet de technicien supérieur agricole) : module du tronc commun «Techniques d'expression, de communication, d'animation et de documentation $"$.

22. F1 = français du premier collectif (M22), F2 = français du deuxième collectif (MG1) et ainsi de suite.

23. Contrôle en cours de formation, une innovation des lycées agricoles datant de 1965.

24. Le module entier repose sur un travail pluridisciplinaire utilisant comme support un thème culturel imposé et qui change tous les deux ans. Le thème concerné par notre enquête est : "La mondialisation des échanges et de la culture ", pour la promotion 2011-2013.

25. Module MG1 : "Langue française, langages, éléments d'une culture humaniste et compréhension du monde ». 
Tableau 1 : Présentation des trois collectifs

\begin{tabular}{|c|c|c|c|c|}
\hline $\begin{array}{c}\text { Collectif } \\
\text { d'enseignants }\end{array}$ & Disciplines & $\begin{array}{l}\text { Liens entre } \\
\text { disciplines }\end{array}$ & $\begin{array}{c}\text { Type } \\
\text { de prescription }\end{array}$ & Objectifs communs \\
\hline $\begin{array}{c}\text { Collectif } 1 \\
\text { Module M22 }\end{array}$ & $\begin{array}{c}\text { BTSA } \\
\text { français ; } \\
\text { documentation ; } \\
\text { ESC ; } \\
\text { économie }\end{array}$ & $\begin{array}{l}\text { Collectif } \\
\text { contraint }\end{array}$ & $\begin{array}{l}\text { Prescription } \\
\text { descendante : } \\
\text { travail autour d'un } \\
\text { thème culturel } \\
\text { imposé }\end{array}$ & $\begin{array}{c}\text { Méthodologie transversale } \\
\text { pour } \\
\text { la maîtrise des } \\
\text { compétences de } \\
\text { communication }\end{array}$ \\
\hline $\begin{array}{l}\text { Collectif } 2 \\
\text { Module MG1 }\end{array}$ & $\begin{array}{c}\text { Bac pro } \\
\text { français ; } \\
\text { histoire- } \\
\text { géographie ; } \\
\text { ESC ; } \\
\text { documentation }\end{array}$ & $\begin{array}{l}\text { Collectif } \\
\text { semi- } \\
\text { contraint }\end{array}$ & $\begin{array}{l}\text { Prescription } \\
\text { discrétionnaire : } \\
\text { mise en œuvre } \\
\text { d'un module } \\
\text { du référentiel }\end{array}$ & $\begin{array}{l}\text { Conception } \\
\text { et réalisation d'une } \\
\text { représentation théâtrale } \\
\text { (projet P.H) }\end{array}$ \\
\hline $\begin{array}{l}\text { Collectif } 3 \\
\text { Collectif libre }\end{array}$ & $\begin{array}{l}\text { Toutes } \\
\text { disciplines: } \\
\text { générales } \\
\text { et scientifiques }\end{array}$ & Collectif libre & $\begin{array}{l}\text { Auto-prescription } \\
\text { d'un chantier } \\
\text { technique } \\
\text { en BTSA }\end{array}$ & $\begin{array}{c}\text { Conception } \\
\text { et réalisation d'un } \\
\text { aménagement paysager }\end{array}$ \\
\hline
\end{tabular}

\section{Résultats de l'enquête}

Notre analyse des trois équipes montre que le rapport entre travail collectif et autonomie collective est variable, suivant d'une part la nature de la tâche, et d'autre part le pouvoir d'agir du collectif sur elle. Nos résultats confortent l'idée que la prescription demeure le principe organisateur de l'activité(Amigues, 2003), conditionnant à la fois la manière dont l'activité est mise en œuvre et son devenir. Nous présentons le travail collectif, de la prescription à l'activité, tout en analysant le rapport entre les deux et le lien qu'ils entretiennent avec l'autonomie résultante.

\subsection{Le pouvoir d’agir du collectif 1 est en lien avec le degré d'appropriation de la tâche}

En ergonomie de l'activité, on considère que la tâche doit être appropriée par le travailleur et mise en œuvre selon l'idée qu'il se fait de la tâche. En d'autres termes, il doit nécessairement y avoir congruence entre la prescription et les normes propres à l'enseignant.

\subsubsection{Impossibilité de s’approprier la prescription}

Dans l'esprit des membres du collectif 1, le module M22 n'est pas «le plus représentatif d'un travail collectif, d'un travail de pluri » (ESC1). En effet, notre enquête révèle des difficultés d'appropriation de la tâche et un sentiment dominant d'insatisfaction chez les membres. L'équipe du M22 ne parvient pas à se constituer en collectif de travail. Les membres n'adhèrent pas à la tâche, qui ne leur apporte aucune satisfaction personnelle et professionnelle. 
Ils soulignent un manque de complémentarité entre les disciplines et un problème d'identification des compétences, particulièrement en français et en ESC. Les compétences à travailler dans les deux disciplines ne sont certes pas identiques, mais nous constatons néanmoins une certaine proximité entre elles: en français (objectif 2), la prescription principale porte sur le discours argumentatif et un travail davantage centré sur la langue. Cependant, le sous-objectif 2.1 (la visée du message à produire, le choix de stratégies et de moyens d'expression adaptés) pourrait aussi bien être traité par un enseignant d'ESC. Pour les membres, il y a un problème de délimitation des champs disciplinaires:

"Le problème avec le M22, c'est que ça fait un peu doublon entre l'ESC et le français; on demande un peu la même chose dans les deux, en fait c'est les référentiels, c'est pas très cadré à ce niveau. » (ESC1)

De même, nous remarquons une grande proximité entre l'objectif 2.3 en français: «l'étudiant doit [...] mobiliser les ressources du code paraverbal et du code non verbal (ressources kinésiques et proxémiques) » et l'objectif 3.1 en ESC : «les signes non verbaux de la communication orale (gestes, [...], signes sensoriels, kinésiques, proxémiques) donne[nt] lieu à un travail spécifique».

Ainsi, les enseignants posent le problème de la cohérence de la prescription. Les objectifs ne s'imbriquent pas d'une manière suffisamment différenciée et, par conséquent, il devient difficile de mettre en œuvre un objectif d'enseignement sans avoir l'impression de répéter ce que son collègue a déjà fait ni se dire que son collègue est aussi tout à fait compétent pour faire le même travail:

«L'ESC et le français, on est hyper redondants... C'est là où c'est une aberration ce M22, c'est que tout le monde se marche un peu dessus... Moi je leur fais écrire un édito, mais tu vas pas me dire qu'ESC elle est pas capable de leur faire écrire un édito? Même D1 peut le faire, et le résumé normalement c'est la Doc qui fait le résumé, et moi donc je le fais, j'ai demandé à D1 sa technique et c'est moi qui le fais... Mais franchement... oui les documentalistes non plus ils n'ont pas besoin de moi... Quand tu lis les référentiels ça se voit hein. Mais surtout le prof de français je ne trouve vraiment pas à quoi il sert. » (F1)

Quant à l'enseignant d'ESC, il indique aussi comment les enseignants "se marchent dessus»:

" On a fait un formatif pour les préparer au CCF oral. Au départ, c'était proposé que moi, j'évalue les supports visuels, l'expression orale, le français, et la recherche pour la doc. Ça c'est pas possible! Quand tu évalues comment les supports visuels sont choisis, exploités, tu regardes forcément le plan! » (ESC1) 


\subsubsection{Impossibilité d'agir sur la tâche}

Le thème culturel imposé au programme, le point commun des disciplines du module, ne facilite pas l'appropriation de la tâche. Deux enseignants sur trois déclarent être peu à I'aise pour travailler sur un thème aussi complexe que la mondialisation sans en avoir un minimum d'expertise:

"Ill y a plein d'arguments économiques à avancer... Ce qui est difficile, c'est que les élèves, ils te mettent parfois en difficulté quand même... Ils te disent, oui la crise des subprimes, et puis machin, ah la la. Mais tu vois, des fois, ils dissertent sur des trucs, je ne peux pas savoir s'ils disent des bêtises ou pas, je ne suis pas à l'aise. » (F1)

«Moi ma place j'ai du mal à la trouver... Le thème qu'on étudie, je ne suis pas à l'aise, et F1, c'est pareil... Si on avait un programme où on pouvait faire un peu de technique, on pourrait leur faire chercher des documents de différents types de jardins, sur des sujets qui les intéressent, ils seraient à fond les élèves, la mondialisation, ils en ont rien à faire, on a un thème imposé et qui les ennuie. L'argent l'année dernière, c'était la cata! » (D1)

Les enseignants mentionnent également que le fait de travailler deux années sur le même thème et avec plusieurs disciplines finit par créer de l'ennui chez les étudiants.

\subsubsection{La prescription est vécue comme une contrainte}

Selon le collectif 1 , il y a un déséquilibre entre les objectifs de formation et les moyens attribués. Les enseignants évoquent une surcharge de travail par rapport au nombre d'heures d'enseignement allouées à chaque discipline. En documentation, il s'agit de construire un projet de médiation documentaire mettant en œuvre des savoirs (objectif 1.1: «Mobiliser les concepts et les techniques nécessaires à une recherche et à un traitement de l'information pertinents ») et des savoir-faire (objectif 1.2: «Mettre en œuvre sa capacité d'organisation et de communication de l'information dans le cadre d'une démarche de médiation documentaire », $\mathrm{DA}^{26}$ 2009).

Pour l'enseignant (D1), c'est non seulement « un travail énorme pour seulement l'équivalent d'une demi-heure de cours par semaine» mais, pour les étudiants, «ils ont l'impression de bosser pour pas grand-chose». Quant à l'enseignant d'économie, il déclare également disposer d'un nombre d'heures insuffisant: "On a l'équivalent d'une heure 30 de cours, donc sur 2 ans ça ferait rien du tout » (E1). Ce qui explique qu'en français (une heure par semaine) par exemple, "personne ne s'est jamais battu au bahut pour récupérer les cours de français en BTS» (D1), alors que par ailleurs les classes de BTS sont les plus convoitées

26. Le "Document d'accompagnement», ensemble d'instructions officielles liées au programme d'études, est un document prescriptif pour l'enseignant. 
en lycée agricole. Les deux enseignants de français et de documentation critiquent la charge de travail imposée: "C'est un travail titanesque, tu dois toi-même tout faire, te documenter sur le thème» (F1).

L'implication demandée est démesurée par rapport aux enjeux: "C'est du boulot, ce CCF. Ça leur [les élèves] demande un travail fou pour un si petit coefficient, Carnet de bord + Revue + Oral. C'est disproportionné! ... Moi, les vacances de Noël c'est de la correction, j'ai 30 dossiers avec des documents différents à lire! ... C'est vachement long! Et en plus ça ne me passionne pas! Je me casse pour rien! »(D1).

L'analyse de l'activité du collectif 1 révèle une appropriation de la tâche a minima, c'est-à-dire que son objectif est simplement la mise en œuvre des enseignements et évaluations pluridisciplinaires (la documentation et le français réalisent un CCF commun, et les quatre disciplines sont regroupées sur l'épreuve terminale ET1). En d'autres termes, les enseignants du module contraints de travailler ensemble le font sans entrain et par obligation, en étant peu motivés et avec un résultat peu satisfaisant à leurs yeux. Par conséquent, en termes organisationnels, nous identifions pour ce cas une sorte de résistance passive au nouveau produit et, sur le plan onto-artefactuel, les sujets expriment un ressenti et un vécu négatifs.

\subsection{Collectif 2, le renouvellement du genre professionnel}

Ce qui distingue notre équipe pédagogique du collectif qui en résulte est l'appropriation de la prescription et sa transformation. Le collectif 2 se distingue du 1 sur deux points essentiels: d'abord par la nature de la prescription elle-même, ensuite par les rapports que les membres entretiennent avec elle.

\subsubsection{La nature de la prescription}

Le MG1 est un module pluridisciplinaire marqué par l'absence d'évaluation conjointe (excepté pour la documentation), mais cela n'a pas toujours été le cas puisque, avant la rénovation du bac professionnel (2010), l'évaluation en CCF était commune. Le prescripteur se contente seulement de poser les bases d'un travail collectif potentiel en regroupant des disciplines complémentaires.

En français, la justification de l'activité se reporte au sous-objectif 1.2: "Mettre en voix (théâtre, poésie) » et «Pratiquer l'écriture d'invention ». Pour l'enseignant, la prescription représente une opportunité: "Pendant mon cours, j'ai pas forcément le temps de faire du théâtre» (F2). En histoire-géographie (objectif 3.1, "Étudier la France aux XIXe et $X X^{e}$ siècles »), le lien avec le travail collectif se fait à travers l'exploration de la vie des ouvriers. En documentation, I'objectif 4.2 («Traiter l'information pour un usage ciblé... ») 
permet une recherche sur le travail des ouvriers d'une papeterie et I'utilisation des moulins à eau au début du XXe siècle.

L'enseignant de documentation contraint par la prescription à une activité collective (thématique du CCF en lien avec la formation) ne montre pas pour autant de la réticence comme c'est le cas en M22. II soutient le projet car il y trouve son intérêt: " C'est spontanément qu'on travaille ensemble. Parce que c'est trop de travail un sujet comme ça. Non en général, j'ai très peu d'heures de documentation, donc moi, ça me sert à faire mon CCF». Quant à l'ESC, la réalisation d'une « production culturelle et artistique » favorisant «l'imaginaire et la créativité » (objectif 2.3) est une prescription liée seulement à la discipline et n'est donc pas une obligation pour les autres membres.

À la différence de la prescription du module M22 issu aussi d'une rénovation ${ }^{27}$, le MG1 laisse aux enseignants plus de marge de manœuvre. Les enseignants de français et d'ESC non contraints par la prescription l'utilisent tout de même comme outil de travail pour mettre en place l'écriture et la mise en scène d'une pièce théâtrale jouée devant public. Ils imaginent également des variantes de l'activité puisque le projet P.H a lieu depuis trois ans et présente à chaque fois une déclinaison différente.

\subsubsection{Les rapports entre acteurs et produit}

Nous englobons dans le rapport au produit (la norme ou les règles prescrites par l'organisation) à la fois la façon dont les acteurs perçoivent celui-ci mais aussi les possibilités offertes par sa transformation. Notre enquête révèle que le travail collectif est conditionné par l'instauration d'un équilibre entre l'intérêt personnel et l'intérêt professionnel. Par intérêt personnel, nous comprenons les raisons pour lesquelles un enseignant effectue ou pérennise une activité collective: reconnaissance auprès de sa hiérarchie, de ses pairs et des élèves, plaisir personnel à renouveler ses routines, et renforcement de l'image de soi lorsque l'activité est considérée comme une réussite. Quant à l'intérêt professionnel, il est lié au gain de temps sur la réalisation des objectifs du référentiel lorsque ceux-ci sont partagés entre enseignants, et lorsque l'activité collective permet d'effectuer les enseignements et évaluations.

À mi-chemin entre les deux types d'intérêt se trouve l'apprentissage au contact des pairs que les enseignants reconnaissent comme facteur important de développement personnel et professionnel. Ainsi, la mise en œuvre des enseignements et évaluations ne suffit pas à elle seule à maintenir l'existence d'un collectif. L'intérêt professionnel doit coïncider avec l'intérêt personnel, et c'est ce qui forge la motivation du groupe. L'enseignant de français (MG1) évoque la satisfaction personnelle dans le travail (« Un plaisir personnel... Mettre en place des projets, réaliser des spectacles, faire faire des choses aux jeunes auxquelles ils ne

27. Rénovation en 2011 du tronc commun (incluant le M22) pour la filière BTSA Aménagements paysagers. 
s'attendent pas», F2), la rupture dans les routines ("C'est quand même sympa de faire d'autres choses dans d'autres situations avec d'autres enseignants, de voir les élèves dans d'autres contextes, sur d'autres thématiques. Changer un peu ton cours classique», F2), et le rapprochement avec les élèves ("Au niveau du lien, ça crée quelque chose», F2). Par ailleurs, la conviction que l'activité collective bénéficie réellement aux élèves contribue à sa légitimité aux yeux des enseignants ("La question n’a pas été ce qu'ils ont appris dans la discipline mais ce qu'ils ont retenu en termes d'expérience», F2).

Le travail du collectif 2 est aussi le résultat d'une reconnaissance d'intérêts mutuels: - d'abord, entre l'ESC et le français: I'enseignant d'ESC utilise le français pour faire écrire aux élèves le script des contes qui sera ensuite théâtralisé, et l'enseignant de français profite du projet ESC (objectif 3.3 du module) pour réaliser un atelier d'écriture collective;

- ensuite, entre l'ESC et la documentation: le travail de recherche fournit l'inspiration pour les contes imaginés par les élèves et permet en même temps à l'enseignant de documentation de réaliser son CCF ;

- puis, entre la documentation et l'histoire-géographie: la collaboration permet à l'histoiregéographie d'exploiter une partie de son programme (les industries locales, objectif 3.1 et objectif 3.2) et, en retour, I'histoire-géographie fournit à la documentation une partie de la thématique de recherche contribuant aux produits documentaires;

- enfin, entre l'histoire-géographie et le français : I'exploitation du thème en histoire-géographie bénéficie au français en permettant aux élèves de comprendre le cadre historique de la production littéraire puisque les périodes d'études coïncident;

- quant à I'ESC, les trois autres disciplines concourent à l'élaboration du CCF basé sur une production culturelle et artistique.

Ainsi, l'activité collective représente dans son ensemble un échange équilibré entre les différents enseignants du module, ce qui peut expliquer sa pérennité.

Notre examen de l'activité révèle une formulation entièrement nouvelle de la tâche avec une marge d'autonomie conséquente. Si le collectif devait mettre en œuvre la tâche prescrite sans d'importantes modifications, nous aurions pu avoir la configuration suivante: quelques séances d'enseignement en classe consacrées à l'écriture d'invention en français, un partenariat entre la documentation et un autre enseignant sur une thématique en lien avec le programme de formation, et, en ESC, une production artistique créée par les élèves et évaluée par l'enseignant seul. Or, le projet P.H dépasse de très loin les attentes du prescripteur. C'est un «projet ambitieux» (ESC2) par son ampleur ${ }^{28}$, sa durée et le nombre

28. Les sorties pédagogiques : centre thermal d'une commune renommée ; écomusée ; musée sur l'énergie produite par les chutes d'eau; journée en bateau le long d'un fleuve traversant une ville et ses communes, en compagnie de quatre intervenants extérieurs dont deux urbanistes du CAUE (Conseil d'architecture, d'urbanisme et de l'environnement) intervenant auprès des élèves sur l'aménagement des berges ; lecture, par un comédien, d'un texte littéraire sur le thème de l'histoire du lieu; centre de la micro et nanotechnologie ; sortie spectacle : Le Songe d'une nuit d'été, de William Shakespeare. 
des intervenants y ayant participé29 ${ }^{29}$. Les activités s'étalent sur l'année, incluent des séances d'enseignement face à la classe, des séances en co-enseignement et des sorties pédagogiques (visites culturelles et fréquentation de salles de spectacle). L'activité a été conçue durant les mois de mars, avril et mai 2011 et a démarré en septembre 2012. Elle comporte plusieurs étapes jusqu'au mois de mars 2012, avec une phase finale sous forme d'une représentation théâtrale en plein air. Les membres permanents du collectif sont ceux présents dans le module, mais d'autres enseignants se sont greffés ponctuellement durant les sorties pédagogiques extérieures.

Pour les acteurs, une appropriation satisfaisante de la prescription rejoint la notion de bonheur au travail. Le contraire serait de s'associer à une activité que l'on ne s'est pas appropriée a priori, avec pour conséquence une faible capacité d'agir sur son travail (Clot, 1999). Dans ce cas, le manque de motivation individuelle pour l'activité collective provoque en retour une difficulté de renormalisation de la prescription. Le travailleur ne parvient pas à agir sur la tâche comme c'est le cas pour certains enseignants du module M22. Un enseignant de la classe mais externe au module ${ }^{30}$ nous livre sa réflexion sur ce sujet:

"On perd beaucoup de temps avec les projets. II faut que les gens aiment ça. Si on doit les forcer, ça fait des profs malheureux. Un prof qui arrive à travailler pour lui est un prof heureux. Si on peut travailler pour soi, c'est le bonheur du prof. Le prof, il faut qu'il fasse ce qu'il aime faire, on ne peut pas l'obliger à faire ce qu'il n'aime pas. »

Ainsi, I'usage de soi par soi dans le travail est aussi essentiel que l'usage de soi par les autres (Schwartz, 2000). Le projet P.H résulte de la capacité du collectif à s'approprier la tâche et à forger son style (Clot, 1999), à exprimer ses idées et à trouver leur traduction en action, construisant ainsi un espace d'autonomie. Cependant, I'autonomie est conditionnée par certains facteurs: la transformation de la tâche doit non seulement correspondre aux normes individuelles (usage de soi par soi), mais également aux normes de l'organisation et aux normes collectives (usage de soi par les autres). Contrairement au cas précédent, ce collectif, loin de montrer de la résistance continue face au changement, s'est au contraire approprié l'artefact, l'a transformé pour qu'il corresponde à ses besoins.

\subsection{Collectif 3 , prescripteur du produit}

Le collectif 3 fonctionne sur le mode coopératif dont une caractéristique majeure est la responsabilité limitée, chaque membre se dédiant à la réalisation de la tâche lui incombant. Le travail est réalisé de façon autonome individuellement avec un membre assumant la fonction de chef de projet (enseignant en économie, E3). Ce collectif regroupe l'ensemble

29. Les partenaires du territoire : mairie ; directeur du centre thermal ; centre culturel de la ville ; école primaire où est implanté le lycée agricole ; Association des sports nautiques (pour la sortie en bateau) ; CAUE (pour l'information sur l'aménagement des berges du fleuve).

30. Le statut de témoin à la marge fournit une perspective supplémentaire sur l'objet d'étude (Miles et Huberman, 2003). 
de l'équipe pédagogique de la classe de BTSA mais porté par un noyau dur de trois enseignants. Le projet M.C porte sur l'organisation d'un concours en conception paysagère ( $1^{\text {re }}$ étape) où le jardin du groupe lauréat est choisi pour être implanté ( $2^{\mathrm{e}}$ étape) sur le site du lycée et présenté au public lors de la Journée portes ouvertes.

\subsubsection{Création de la tâche ou auto-prescription}

Si les compétences en lien avec le projet M.C se retrouvent bien dans le référentiel professionnel ${ }^{31}$, en revanche la réalisation d'un chantier d'aménagement n'est pas obligatoire. La conception et l'aménagement paysager peuvent donc être considérés comme significativement divergents. Si l'adhésion au projet présente un caractère non contraignant, toutefois, à partir du moment où le collectif est établi et ses membres identifiés, le plan mis en place agit comme une sorte de contrat implicite entre les membres qui s'engagent à le respecter. C'est pour cette raison que nous considérons l'accord implicite sur le projet comme une sorte d'auto-prescription. Nous avons ainsi qualifié le groupe de collectif libre puisqu'il n'est lié par aucune prescription externe. Aussi, le projet étant technique, il n'est pas surprenant de constater sa proximité avec le référentiel de formation. Nous constatons en effet un lien étroit entre le projet et plus de la moitié des enseignements du référentiel professionnel${ }^{32}$.

À l'origine du projet est la proposition pour la classe de BTSA Aménagements paysagers (2011-2013) de participer à un festival international de jardins. Pour 2012, le thème est "Jardins des délices, Jardins des délires», avec des œuvres exposées pour une durée de six mois, d'avril à octobre 2012. L'esprit du festival est la présentation de jardins « originaux, novateurs, audacieux ou oniriques» (site internet du festival). Devant l'ampleur de la préparation et le peu de temps disponible car il s'agissait de travailler avec la promotion entrante, les membres ont jugé qu'il était impossible pour eux $d^{\prime}$ " être dans les clous» (E3) par rapport aux échéances imposées par le festival.

Ainsi, ils ont abandonné l'idée de participation au concours au profit d'une compétition locale, le projet M.C, où finalement ce sont les étudiants entre eux qui ont concouru pour la meilleure conception de jardin mais toujours sur le thème des «Jardins des délices, Jardins des délires ». Cependant, le voyage d'étude a été maintenu et la classe s'est rendue au Festival des jardins avec quatre enseignants ${ }^{33}$ en septembre 2011. L'objectif principal était que les étudiants voient les jardins exposés et se familiarisent avec l'esprit du festival dont les œuvres sont conceptuelles et non classiques. Une fois le jardin conçu et réalisé sur le site de l'établissement, il fut présenté devant le maire de la commune et d'autres

31. Exemples : objectif du module D3.2 : "Calcul de coûts sur un chantier pour les techniques en sciences économiques" ; objectif I du module D4.3 : "Création plastique pour la conception en aménagement ». Extraits du "Référentiel professionnel du Technicien supérieur en Aménagements paysagers ", DGER, janvier 1994.

32. Référentiel professionnel, BTSA, arrêté du 28 octobre 1993. II s'agissait encore du BTSA non rénové.

33. Deux enseignants techniques, un de français et une artiste plasticienne. 
personnalités locales ainsi que des journalistes. Des plaquettes explicatives du projet furent aussi conçues par les élèves et distribuées au public. Le projet, reflétant le principal enseignement de spécialité, est considéré comme une vitrine pour l'établissement.

\subsubsection{Expérimentation avec le genre professionnel}

Le travail collectif dans notre troisième cas aboutit à une expérimentation avec le genre professionnel. Les enseignants imaginent une variante de l'activité, la testent et se livrent à une réflexion collective a posteriori pour évaluer à la fois le processus et le résultat de l'activité collective. Contrairement aux deux autres collectifs dont l'activité émane d'une source externe, le projet M.C est une activité auto-prescrite. Par conséquent, certaines divergences dans le mode opératoire sont visibles: alors que les deux collectifs suivent les étapes habituelles du processus de l'activité (interprétation de la prescription et mise en place de l'activité), M.C en représente une extension en englobant des responsabilités spécifiques relatives à la tâche et au devenir de l'activité. En effet, le collectif conçoit et réalise lui-même la tâche et cette initiative lui donne un pouvoir d'agir sur la manière de la réaliser. II devient ainsi l'instrument de sa propre action, ce qui explique un élargissement de la marge d'autonomie associée à l'appropriation de la tâche.

L'expérimentation avec le genre professionnel dans le cas du collectif 3 englobe aussi une étape réflexive où les «failles» du projet sont identifiées et les possibles (le non-réalisé, Clot 1999) de l'activité discutés. Lors d'un retour sur l'activité, les enseignants du collectif mentionnent plusieurs points.

D'abord, c'était une expérience difficile en termes de charge de travail: "C'est fatiguant, épuisant pour les personnes qui mènent le projet, enfin, on était trop peu nombreux à gérer » (T3-1). «Pendant le chantier, j'ai passé deux nuits à pas dormir, le stress était quand même intense. Il fallait tout faire, c'était trop de trucs à gérer» (T3-2).

Ensuite, I'un des trois membres évoque le manque d'anticipation par rapport à l'aspect technique du chantier: "Il y a eu des erreurs techniques, les bordures, c'est vrai que ça se dilate énormément à la chaleur, c'est pas parfaitement rectiligne par rapport à ce qu'on aurait souhaité, la tête du géant eh ben, ça commence à s'écarter un peu » (T3-1).

Puis, le manque d'anticipation par rapport au planning: "Le chantier, je me suis retrouvé souvent à tout gérer, 20 étudiants sur le chantier des fois, c'était dur »(T2).

Le coordonnateur, quant à lui, juge que M.C est un projet à valeur d'essai: "Justement, c'est en faisant le projet que tu bouscules un peu le truc..., tu es obligé de t'adapter, tu vois qu'est-ce qui prend du temps, qu'est-ce qui prend pas de temps. Ouais, bien sûr les erreurs que tu fais, comment il faut faire autrement... »(E3). Malgré les faiblesses du 
projet, il était utile de le faire pour «montrer que c'était possible, qu'on pouvait le faire avec une classe, que ça fonctionnait».

Par ailleurs, la réflexivité des membres s'oriente aussi sur l'enseignement: « Le projet est complexe, on leur demande d'intellectualiser alors qu'ils débutent leur formation. Certains étudiants étaient décontenancés; c'était trop conceptuel pour eux. On a rapidement vu la difficulté, il y en avait un qui disait, ça sert à rien ce truc; pour les étudiants comme ça, un jardin, c'est forcément avec des plantes, ceux-là n'ont pas aimé » (T3-2). Pour le coordonnateur, le projet M.C permet aussi d'expérimenter de nouvelles pratiques d'enseignement: "On peut dire que... pour faire M.C, ils ont besoin d'acquérir des compétences en amont. L'idée, c'est d'arriver à les faire travailler de façon plus autonome et qu'ils aillent eux-mêmes en recherche d'informations. Comment on fait pour faire un muret? Et l'idée, c'est qu'ils aient des ressources de cours qui s'adaptent à ça. Ce genre de projet, ça remet en cause complètement notre façon de fonctionner... Voilà, c'est très riche personnellement»(E3).

Le collectif 3 est parvenu à créer un espace d'échange et de partage d'idées permettant de redynamiser le travail en renouvelant les routines. II a également contribué à un environnement d'apprentissage social et à la possibilité de mieux faire face à la complexité de la tâche. Nous constatons aussi une congruence entre les normes des enseignants et I'activité au travers de la reconnaissance sur le plan pédagogique d'un projet liant apports théoriques et techniques tout en créant pour les étudiants la rencontre avec les aléas du métier.

Le collectif 3 est celui qui se rapproche le mieux du changement onto-épistémologique. L'activité inédite a en effet provoqué des réflexions partagées et une réflexivité des sujets sur l'action: même si celle-ci ne se révèle pas entièrement satisfaisante à leurs yeux, les possibles prennent cependant de l'ampleur puisqu'ils permettent de construire un capital de connaissances personnelles et collectives diffusables à tout moment. Les membres, en devenant auteurs du changement et prescripteurs de l'artefact, créent avec lui une proximité telle que ce dernier finit par s'inscrire dans leur histoire et devient un outil de développement du sujet.

\section{Conclusion}

À travers l'analyse du travail de nos trois équipes d'enseignants, nous identifions plusieurs plans de changement: d'abord, sur un plan onto-artefactuel, nous venons d'explorer comment les sujets collectifs de l'organisation scolaire interagissent avec le produit: la prescription, comment ils la reçoivent et la perçoivent (collectifs 1 et 2), et comment ils la façonnent (collectifs 2 et 3 ) pour en faire un objet toujours en changement. Ensuite, nous constatons que les acteurs de la prescription remontante (collectifs 2 et 3 ) participent à un flux continu de changement complexe dans le temps, puisqu'ils font partie de collectifs 
projetant les sujets en action (plan onto-praxique) et mettant en jeu des connaissances et des savoirs dont la diffusion est possible à travers l'organisation (plan onto-épistémique). Enfin, nos trois collectifs sont concernés par le plan arte-praxique puisque, à travers leurs actions, ils font évoluer la prescription et la transforment. C'est pourquoi les pratiques humaines professionnelles diffèrent tant d'un lieu à un autre, car non seulement elles sont le produit d'interactions uniques à un instant $t$ mais, comme nous l'avons mentionné plus haut, le contexte organisationnel présente aussi un élément déterminant dans la caractérisation et la pérennisation du genre professionnel.

Quant aux résultats du changement, les prescriptions successives encourageant la pluridisciplinarité sont venues renforcer une pluridisciplinarité déjà existante dans les lycées agricoles. Au travers de l'exploitation directe ou indirecte des référentiels, les équipes pédagogiques se construisent un espace d'autonomie pour concevoir et mettre en œuvre les enseignements. L'exemple de nos trois équipes renforce l'idée d'une nouvelle configuration du travail enseignant (Marcel, 2006), où le travail collectif occupe désormais une part significative. Issu à la fois de sources descendante et ascendante, le changement que constitue le travail collectif des enseignants affecte trois types d'acteurs: I'établissement, les équipes d'enseignants et les élèves. Pour l'établissement, il représente un potentiel de développement en capitalisant sur les gains cognitifs de ses membres et contribuant à son rayonnement sur le territoire. Pour les enseignants, il signifie un espace d'autonomie où s'exprime la création professionnelle et, pour les élèves, il est source potentielle d'expériences enrichissantes.

Nous venons de voir comment un changement prescrit par l'organisation, la pluridisciplinarité introduisant de fait le travail collectif, est finalement approprié par les équipes pédagogiques, lesquelles ne se contentent pas simplement de l'appliquer, mais dépassent les attentes du prescripteur en s'auto-prescrivant des modules pluridisciplinaires là où ils n'existent pas. Cependant, la pérennisation d'un collectif de travail est soumise à l'existence d'un équilibre essentiel entre intérêts personnels et professionnels, entre « l'usage de soi par soi et l'usage de soi par les autres»(Schwartz, 2000). 